\section{EVALUATING A NURSE LED TRIAGE PROCESS IN TREATING PATIENTS WITH LEFT BUNDLE BRANCH BLOCK (LBBB) REFERRED FOR PRIMARY PERCUTANEOUS CORONARY INTERVENTION (PPCI)}

doi:10.1136/heartjnl-2011-300198.10

${ }^{1} \mathrm{~N}$ V Joshi, ${ }^{2} \mathrm{~B}$ R Bawamia, ${ }^{2} \mathrm{~S}$ Jamieson, ${ }^{2} \mathrm{~A}$ Zaman, ${ }^{2} \mathrm{R}$ Edwards. ${ }^{1}$ Centre for Cardiovascular Science, The University of Edinburgh, Edinburgh, UK; ${ }^{2}$ The Cardiothoracic Centre, Freeman Hospital, Newcastle Upon Tyne, UK

Background The Freeman Hospital (FRH) performs over 900 pPCI a year. Patients with suspected Acute Myocardial Infarction (AMI) are referred either by paramedics or networked hospitals for consideration of pPCI via a Telmed system, which is triaged by experienced CCU nurses. The pPCI Pathway can be activated in patients with LBBB suspected of having an AMI. However, there remains considerable variation in the clinical utility of new or presumed new LBBB as a ST-elevation myocardial infarction (STEMI)-equivalent ECG diagnostic criterion. The major discriminators the triage staff use in this population are ECG findings and symptoms suggestive of AMI. Our aim was to evaluate outcomes in patients with LBBB accepted to FRH or referred to local hospitals for assessment.

Methods Consecutive patients referred to FRH with LBBB and suspected AMI from 1st August 2009 to 30th November 2009 were analysed by recording: 1) Peak Troponin Level 2) Angiographic findings 3) Revascularisation rates.

Results 1069 patients were referred for consideration of pPCI. 177 $(16.6 \%)$ of patients had new or presumed new LBBB. $33(18.6 \%)$ patients were accepted by FRH and 144 patients (81.4\%) were declined and referred to their local hospitals for assessment. Abstract 10 Table 1 Troponin levels in patients with LBBB referred for consideration of pPCI. $26.5 \%$ of patients with $\mathrm{LBBB}$ referred for consideration of pPCI had moderately to highly raised troponin. Of the 33 patients admitted to FRH, 13 underwent inpatient angiography and 9 patients had significant coronary disease (coronary stenosis $70 \%-100 \%$ in at least one coronary artery). Of those, 5 had PCI and 1 required urgent CABG. Only one patient had a $100 \%$ coronary occlusion believed to be an acute occlusion. 4 patients had unobstructed coronaries and were managed medically. Of the 132 patients declined for pPCI only $2(1.5 \%)$ were referred back to FRH for PCI. Neither of these patients was found to have a $100 \%$ acute occlusion of a coronary artery.

\section{Abstract 10 Table 1}

\begin{tabular}{|c|c|c|}
\hline & FRH Assessed & FRH Declined \\
\hline Number of patients & 33 & 144 \\
\hline Number analysed & 33 & $\begin{array}{l}132 \text { (Biochemistry } \\
\text { data not found for } \\
12 \text { patients) }\end{array}$ \\
\hline \multicolumn{3}{|l|}{ Troponin levels } \\
\hline Trop $\mathrm{I}<0.04$ or Trop $\mathrm{T}<0.01$ (Normal) & $19(57.6 \%)$ & $84(63.6 \%)$ \\
\hline Trop I $0.04-0.1$ or Trop T $0.01-0.1$ (Mildly raised) & $2(6.1 \%)$ & $25(18.9 \%)$ \\
\hline Trop I or Trop T $0.1-1.0$ (Moderately raised) & $1(3.0 \%)$ & $17(12.9 \%)$ \\
\hline Trop I or Trop T>1.0 (High) & $11(33.3 \%)$ & $6(4.5 \%)$ \\
\hline
\end{tabular}

Conclusion Revascularisation was performed in only 6/33 (18.2\%) accepted for assessment and only 2/132 (1.5\%) were referred back to the centre for PCI. The sensitivity of the triage process in detecting patients with $\mathrm{LBBB}$ requiring urgent revascularisation is $75 \%$ and the specificity is $83 \%$. The sensitivity of detecting patients with an acutely occluded artery diagnosed at angiography is $100 \%$ with a specificity of $81 \%$. In a high volume Heart Attack Centre a nurse led triage is effective at discriminating patients with $\mathrm{LBBB}$ requiring immediate coronary intervention.

\section{COPEPTIN IMPROVES EARLY RISK STRATIFICATION BY GRACE SCORE IN NON ST-ELEVATION MYOCARDIAL INFARCTION; NT-PROBNP DOES NOT}

doi:10.1136/heartjnl-2011-300198.11

${ }^{1} \mathrm{O}$ S Dhillon, ${ }^{1} \mathrm{H}$ K Narayan, ${ }^{1} \mathrm{P}$ A Quinn, ${ }^{2} \mathrm{~J}$ Struck, ${ }^{1} \mathrm{I}$ B Squire, ${ }^{1} \mathrm{~J}$ E Davies, ${ }^{1} \mathrm{~L} \mathrm{~L}$ Ng. ${ }^{1}$ University of Leicester, Leicester, UK; ${ }^{2}$ Brahms, Hennigsdorf, UK

Background Risk stratification is vital to the optimal management of patients with non ST-elevation myocardial infarction (NSTEMI) however, current tools are not fully discriminatory. Copeptin, the stable 39 amino acid C-terminal portion of pro-vasopressin, is a recognised prognostic marker in ST elevation myocardial infarction (STEMI) that is also useful to exclude MI as levels rise early after onset. Copeptin has not been evaluated in a NSTEMI population to date.

Aims We hypothesised that copeptin is an independent predictor of mortality following NSTEMI and, in accordance with AHA criteria for the evaluation of novel biomarkers, assess whether copeptin adds prognostic information to GRACE risk score (GRACE-RS). We use NT-proBNP for comparison.

Methods and Results In this prospective observational study plasma copeptin and NT-proBNP was measured in 754 NSTEMI patients (519 men, median age $70 \pm 13$ years) within $36 \mathrm{~h}$ of symptoms. The primary endpoint of all-cause mortality at 6 months was reached by $56(7.4 \%)$ patients. Median copeptin levels were 7.9 range 0.3 to $523.0 \mathrm{pmol} / 1$ and were significantly higher in those that reached the primary endpoint than the event free survivors; median (IOR), 32.0 $(12.0-88.7)$ vs $7.2(4.0-16.7)$ respectively $p<0.001$. Both copeptin and NT-proBNP were predictive of the primary endpoint on univariate Cox regression analysis (HR 5.98 p $<0.0005$ and HR 6.07 p $<0.0005$ respectively). On adjustment for baseline clinical and biochemical variables copeptin remained predictive (HR $3.03 p=0.009)$ but NTproBNP did not $(p=0.70)$. Kaplan-Meier analysis revealed that supramedian levels of copeptin were associated with increased mortality (log rank $28.4 \mathrm{p}<0.001$ ). ROC curve c-statistics for GRACE-RS of 0.799 increased to 0.835 when combined with copeptin (0.785), when combined with NT-proBNP (0.730) increased to 0.802. Re-classification analysis shows that copeptin improves accuracy of risk stratification when combined with the GRACE-RS as determined by net reclassification improvement (NRI 13.3\% $\mathrm{p}=0.008$ ) whereas, NTproBNP does not (NRI $-4.9 \% \mathrm{p}=0.21$ ). The relative utilities for logistic regression models using GRACE-RS alone, GRACE-RS + copeptin and GRACE-RS + NTproBNP as covariates are shown in Abstract 11 figure 1 . The relevant region was the region to the right of the sample risk for 6 months mortality, 0.074. The relative utility for GRACE-RS + copeptin was consistently more than the relative utility for GRACE-RS + NT proBNP across a range of risks; for example at a risk threshold of $15 \%$ the additional utility of adding copeptin to the GRACE-RS was 0.097 compared to 0.009 for NTproBNP.

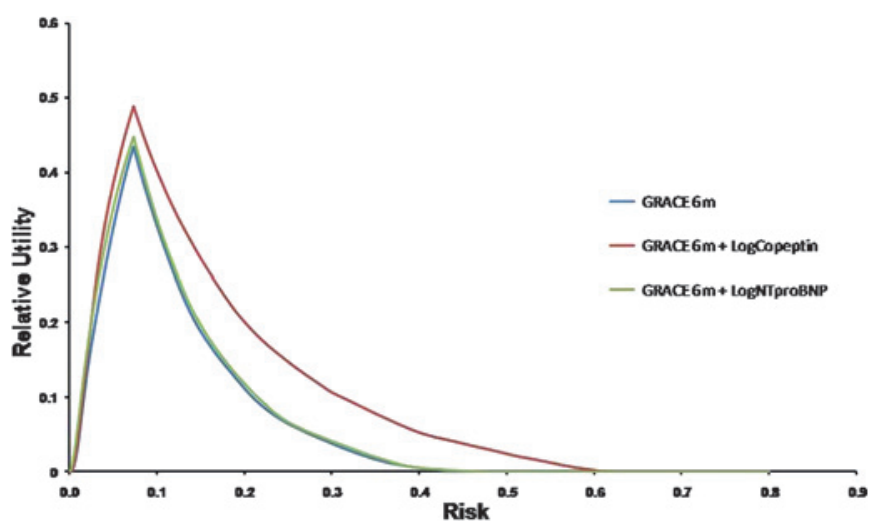

Abstract 11 Figure 1 
Conclusions High plasma copeptin levels indicate a worse outcome in NSTEMI patients. We have demonstrated that copeptin fulfils AHA criteria by improving risk stratification over established markers GRACE score and NT-proBNP. Copeptin is also useful for rapid rule-out of $\mathrm{MI}$ and the current findings further support clinical uptake.

\section{THE RELATIONSHIP BETWEEN PSYCHOLOGICAL FACTORS AND IMPAIRED HEALTH-RELATED QUALITY OF LIFE POST ST-ELEVATION MYOCARDIAL INFARCTION}

doi:10.1136/heartjnl-2011-300198.12

${ }^{1} \mathrm{~L}$ McGowan, ${ }^{2} \mathrm{H}$ lles-Smith, ${ }^{1} \mathrm{C}$ Dickens, ${ }^{1} \mathrm{M}$ Campbell, ${ }^{1} \mathrm{C}$ Rogers, ${ }^{2} \mathrm{~F}$ Fath-Ordoubadi. ${ }^{1}$ University of Manchester, Manchester, UKI; ${ }^{2} \mathrm{CMFT}$, Manchester, UK

Introduction Evidence suggests that psychological factors, such as depression and anxiety, are independent risk factors for increased morbidity and mortality post ST-elevation myocardial infarction (STEMI). Since improved treatments have increased survival rates post STEMI the emphasis has turned to more patient related outcome measures such as health-related quality of life (HRQoL). The aim of the study was to assess the contribution of anxiety and depression to HRQoL in post STEMI patients, after controlling for possible confounding factors, including type of treatment.

Methods We conducted a prospective cohort study of 385 postSTEMI patients who had undergone either lysis (183) or PPCI (202). The mean age was 60.0 years (SD 11.8) and $78 \%$ were male. Patients were assessed on a range of demographic, clinical and psychosocial variables, including measures of cardiac risk, cardiac severity and comorbidity (Charlson Comorbidity IndexCCI). Psychosocial assessment included anxiety and depression (Hospital Anxiety and Depression Scale), illness perceptions (brief IPQ), and health-related quality of life (SF-36). The main outcome was the SF-36 Physical Component Score (PCS) at 6 months postSTEMI

Results Baseline results revealed a small number significant differences between groups on a range of clinical variables, including higher GRACE scores for PPCI group $(p=0.007)$ but no differences in LV function. Lysis patients had more comorbid illness as measured by the CCI ( $p=0.037)$. Regarding psychological variables the total HADS score was significantly higher in the PPCI vs lysis group at baseline (means 13.2 (SD 7.9) and 11.4 (SD 8.9), $\mathrm{p}=0.035$ ), while anxiety and depression almost reached significance, with raised anxiety and depression scores in the PPCI group. In order to identify variables at baseline that may contribute to SF-36 PCS at 6 months, we conducted a hierarchical multiple regression with four blocks of independent variables-demographic, comorbidity-related, clinical and psychological. Factors which contributed to the final model were cholesterol levels $(p=0.031)$ and depression $(p<0.001)$. Treatment group did not play a role $(\mathrm{p}=0.199)$. The addition of anxiety and depression contributed significantly to the reporting of lower physical health-related quality of life (PCS) at 6 months ( $\ddot{\mathrm{A}} 2=0.12$, $\mathrm{p}<0.001$ ).

Conclusion The findings have shown that raised levels of depression and anxiety predicted impairment in health-related quality of life at 6 months post-STEMI, regardless of mode of treatment. The results indicate that the assessment of psychological factors is important in both groups. Despite PPCI having improved clinical outcomes, there will always be a group of patients receiving lysis. As such it is important to assess anxiety and depression in post STEMI patients, and to include these potentially modifiable factors in the design of suitable interventions for this patient group.

\section{NEUTROPHIL ACTIVATION AT THE CULPRIT LESION IN ACUTE ST-SEGMENT ELEVATION MYOCARDIAL INFARCTION WITH MULTIPLE COMPLEX CORONARY PLAQUES}

doi:10.1136/heartjnl-2011-300198.13

${ }^{1} \mathrm{C} \mathrm{J}$ Marshall, ${ }^{1} \mathrm{~J}$ L Mckenzie, ${ }^{2} \mathrm{~T}$ Moccata, ${ }^{3} \mathrm{M}$ Nallaratnam, ${ }^{3} \mathrm{~J}$ Blake, ${ }^{3} \mathrm{C}$ Frampton, ${ }^{3} \mathrm{M}$ Richards, ${ }^{2} \mathrm{~A} J$ Kettle, ${ }^{3} \mathrm{D}$ R Mcclean. ${ }^{1}$ Sunderland Royal Hospital, Sunderland, UK; ${ }^{2}$ Free Radical Research Group, University of Otago, Christchurch, New Zealand; ${ }^{3}$ Department of Cardiology, Christchurch Hospital, Christchurch, New Zealand

Introduction The activation of neutrophils at the culprit coronary lesion following acute plaque disruption has not been reported. We hypothesised that neutrophil activation occurs in ST elevation myocardial infarction (STEMI) prior to percutaneous intervention (PCI), and that differences in activation may be detectable locally at the culprit lesion, particularly in patients with multiple complex coronary plaques.

Methods Forty STEMI patients having primary PCI were compared to 10 controls with chronic stable angina (CSA) undergoing elective PCI. The clinical, demographic and angiographic characteristics of patients and controls are shown in Abstract 13 table 1 . The culprit lesion was sampled after passage of a guide wire across the lesion and use of a low profile sampling catheter (Multifunctional probing catheter, Boston Scientific Corporation, Natick, Massachusetts, USA) at the site of occlusion, prior to further mechanical intervention. Neutrophil activation was measured by flow cytometry using neutrophil intracellular myeloperoxidase content (MPO Index) and the expression of the $\beta 2$ - integrin CD11b, a leukocyte adhesion and activation marker at the culprit coronary lesion (CA), the aorta at the coronary ostium (Ao), the coronary sinus (CS), and femoral artery (FA) prior to primary PCI. A lower $\mathrm{MPO}$ content indicates the depletion of intracellular MPO and cell activation.

\section{Abstract 13 Table 1}

\begin{tabular}{lllc}
\hline Variable & $\begin{array}{l}\text { STEMI } \\
(\mathbf{n = 4 0 )}\end{array}$ & $\begin{array}{l}\text { Elective PCI } \\
(\mathbf{n = 1 0})\end{array}$ & $\mathbf{p}$ Value \\
\hline Age (years) mean \pm SD & $62 \pm 12$ & $68 \pm 9.1$ & 0.9 \\
Male (\%) & $28(70)$ & $7(70)$ & 1 \\
$\begin{array}{l}\text { Culprit coronary artery lesion treated (\%) } \\
\text { Left anterior descending }\end{array}$ & $17(42)$ & $5(50)$ & 0.73 \\
Diagonal & $2(5)$ & $1(10)$ & 0.5 \\
Circumflex & $1(3)$ & $2(20)$ & 0.1 \\
Right coronary & $20(50)$ & $2(20)$ & 0.15 \\
Time to presentation (mins) mean $\pm S D$ & $222 \pm 155$ & NA & \\
Baseline TIMI flow 0-1 & $28(70)$ & 0 & $<0.001$ \\
\hline
\end{tabular}

Results A marked decrease in MPO content occurred at the CA, Ao and FA in STEMI compared to elective controls $(p<0.01)$. Furthermore, MPO content was lower at the CA $(-23.1,(-25.6$ to -17.1$)$, $\mathrm{n}=37)$ compared to Ao $(-22.0,(-24.7$ to -16.2$), \mathrm{n}=37)$, CS $(-20.6$, $(-24.8$ to -16.9$), \mathrm{n}=30)$ and FA $(-20.4,(-24.4$ to -13.1$), \mathrm{n}=40)$, all $\mathrm{p}<0.001$ (Abstract 13 figure 1). Neutrophil MPO content was correlated with CD11b expression only at the culprit CA in STEMI $(\mathrm{r}=-0.4, \mathrm{p}=0.03, \mathrm{n}=31$ ) (Abstract 13 figure 2). Neutrophil MPO content at the CA in patients with multiple complex plaques was similar to those with a single culprit however only in those with multiple complex plaques was a correlation between MPO content and CD11b ( $\mathrm{r}=-0.7, \mathrm{p}=0.02)$ shown. Conclusion: In acute STEMI, neutrophils are activated systemically, regionally and locally at the culprit coronary lesion. In patients with multiple complex plaques, there may be an extended local role for the activated neutrophil following acute plaque destabilisation. 